\title{
ESTUDO DO ESCOAMENTO BIFÁSICO EM UM DUTO COM VAZAMENTOS MÚLTIPLOS EM REGIME PERMANENTE E TRANSIENTE
}

\author{
J.W.S. COSTA ${ }^{1}$, P.H.C. DE MELO ${ }^{1}$, J.V.O. MUNIZ ${ }^{1}$, F.F. de OLIVEIRA ${ }^{1}$, W.G. VIEIRA ${ }^{1}$, \\ C.H. SODRÉ ${ }^{1}$ \\ ${ }^{1}$ Universidade Federal de Alagoas, Centro de Tecnologia \\ E-mail para contato: jamersonwillian94@gmail.com
}

RESUMO - O transporte bifásico de líquido e gás através de tubulações está presente em diversas situações na indústria química e petroquímica. Vazamentos em tubulações podem ocorrer devido a uma série de fatores como corrosão, fadiga mecânica, falhas de soldagem, mudanças bruscas de pressão entre outros. O presente estudo tem como objetivo analisar as características dos perfis de escoamento bifásico (água-gás) isotérmico em regime estacionário e transiente em tubos horizontais na presença de vazamentos. Para isto foi usado o software ICEM presente no ANSYS para a geração das malhas estruturadas. Foram usados os modelos Eulereano-Eulereano e o de turbulência k- $\varepsilon$ junto com as equações da continuidade, de momento e de energia para resolução do problema proposto através do software ANSYS-CFX. Dois pontos de vazamentos distintos foram simulados ao mesmo tempo ao longo da tubulação. Os comportamentos das variáveis de pressão, velocidade e fração volumétrica do fluido foram avaliados a fim de adquirir os perfis característicos para este tipo de problema. Quedas de pressão decorrente do vazamento foram observadas.

\section{INTRODUÇÃO}

O estudo do vazamento visa à identificação dos perfis e características dos mesmos no escoamento de determinado fluido ou em misturas. Sua importância está ligada obviamente a perda de material através do vazamento, possíveis perdas humanas e desastres ambientais que acarretam em multas de valores na casa dos milhões, além de gerar insegurança à população. A presença de vazamento no escoamento em dutos é algo frequente, que deve ser identificada de forma rápida e precisa a fim de diminuir os danos financeiros e ambientais. Os vazamentos podem surgir como consequência de corrosão, fadiga mecânica, falhas de soldagem, mudanças bruscas de pressão, entre outros (Colombo et al., 2009). Para isto faz-se o uso de detectores de vazamento, que utilizam diversos princípios físicos para tal função. Sua aplicação varia com as condições a que se encontram os dutos e os fluidos que são transportados, sendo o mais simples o balanço de massa e a modelagem computacional (Colombo et al., 2009), proposta neste estudo.

As companhias de abastecimento sofrem com este problema pelo fato de suas tubulações serem de difícil manutenção e quase nunca possuem sistemas de detecção de vazamentos. A presença de vazamentos se torna ainda mais preocupante quando o fluido transportado é tóxico e/ou de alto valor 

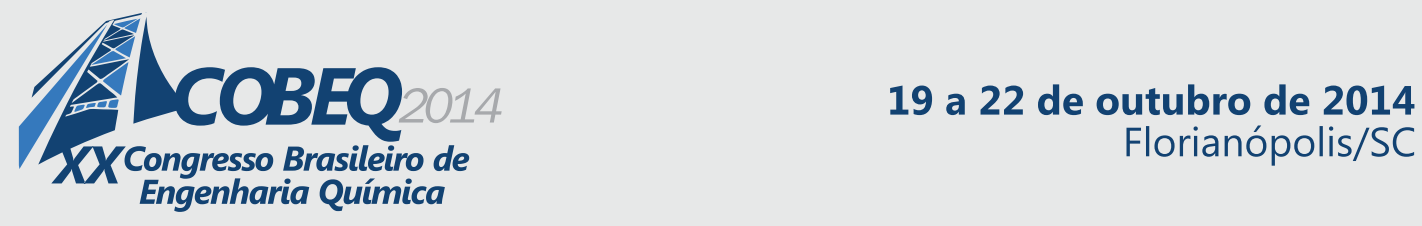

agregado, o que ocorre no transporte de gases, ácidos e hidrocarbonetos mais comumente nas operações industriais. Mesmo com os problemas que podem ser gerados pela presença de vazamento, a maioria das empresas não para o processo para que o problema seja solucionado e apenas uma pequena parte utiliza de dispositivos para detectar vazamento, como indica as pesquisas da PHMSA Pipeline and Hazardous Materials Safety Administration. Um dos fatos para a não utilização dos detectadores de vazamentos pelas empresas se dá pela falta de precisão no processo de detecção gerando falsos alarmes de vazamentos, pelo alto custo agregado em alguns, e pelo problema da instalação em alguns casos específicos. A melhora de tais processos se torna crucial para que o seu uso seja adotado e que isto seja possível se faz necessário o conhecimento do processo que está sendo estudado.

Diversos trabalhos são encontrados na literatura, onde se propõe a simulação de vazamentos, como um método importante para a prevenção de falhas e estudo do perfil do escoamento (Souza, 2010). Sendo então a simulação computacional como uma técnica a mais que visa complementar as análises teóricas e experimentais para que possa ter um melhor esclarecimento do processo (Bakker, 2002; Korkischko, 2006; Bigarella, 2007; Marinho, 2008). Vale ressaltar que a simulação computacional é imprescindível no estudo do processo quando não é possível resolução teórica analítica do processo, como no caso do estudo da aerodinâmica (Lomax et al., 1999), sendo estas equações diferenciais não-lineares que podem ser resolvidas com condições de contorno adequadas (Santos, 2010).

Neste artigo optou-se então pelo estudo do escoamento de água e gás através de dutos tubulares com presença de vazamento. Foi estudado então o escoamento em regime permanente e transiente. Para a modelagem do processo foi utilizado o software ANSYS-CFX. Para a análise do resultado obtido fez-se o uso das curvas de pressão, velocidade e fração volumétrica dos componentes; tendo como base de validação as características descritas na literatura.

\subsection{METODOLOGIA}

O padrão de escoamento adotado no presente estudo foi o de bolhas, onde a fase contínua é a água e a fase dispersa é o ar. Para definição do padrão de fluxo foi utilizado o mapa de fluxo construído por Backer (1954) que pode ser encontrado no Perry's Handbook (6 $6^{\mathrm{a}}$ Ed, 2008). Obteve-se então os valores de fluxo de massa por unidade área de gás de $2,423 \mathrm{~kg} / \mathrm{m}^{2} \mathrm{~s}$ e fluxo de massa por unidade de área de líquido de $5002 \mathrm{~kg} / \mathrm{m}^{2} \mathrm{~s}$.

Para o cálculo da fração volumétrica de líquido foi utilizado o gráfico desenvolvido por Lockhart e Martinelli (1949), que relaciona um parâmetro X a fração volumétrica de líquido que é função apenas da perda de carga de cada fase. A equação para X e o gráfico está representada pela equação (1) e (2).

$$
\begin{aligned}
& \mathrm{X}=\left[(\Delta \mathrm{P} / \Delta \mathrm{L}) \mathrm{l} /\left([(\Delta \mathrm{P} / \Delta \mathrm{L}) \mathrm{g}]^{0,5}\right.\right. \\
& (\Delta \mathrm{P} / \Delta \mathrm{L}) \mathrm{f}=\left(\rho \mathrm{f}^{*} \mathrm{jf}^{*} \mathrm{D}_{\mathrm{i}}\right) / \mu \mathrm{f}
\end{aligned}
$$

Sendo $(\Delta \mathrm{P} / \Delta \mathrm{L}) 1$ a perda de carga da fase líquida por unidade de comprimento, $(\Delta \mathrm{P} / \Delta \mathrm{L}) \mathrm{g}$ a perda de carga da fase gasosa por unidade de comprimento, $(\Delta \mathrm{P} / \Delta \mathrm{L}) \mathrm{f}$ a perda de carga da fase em questão, $\mu \mathrm{f}$ 


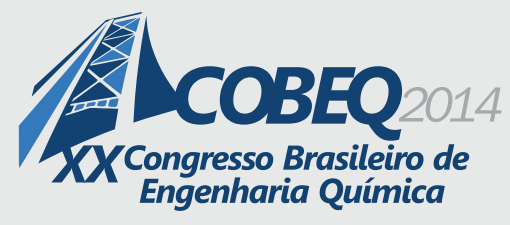

19 a 22 de outubro de 2014
Florianópolis/SC

a viscosidade dinâmica do fluido na fase, $\rho$ f a massa específica da fase, $\mathrm{j}$ f a velocidade superficial da fase e $\mathrm{Di}_{\mathrm{o}}$ diâmetro interno do tubo.

Após o cálculo e a consulta do gráfico foi possível chegar a um valor aproximado para a fração volumétrica de líquido de 0,82 , consequentemente, a fração volumétrica de gás é de 0,18.

Para definir o diâmetro médio das bolhas foi usada a correlação de Hinze (1976), equação (3), para escoamento do tipo bolha dispersa de gás em líquidos por tubos horizontais em que descreve o valor máximo que essas bolhas podem chegar antes de deixar a fase dispersa e começar o processo de coalescência das bolhas. Por ser o diâmetro máximo possível, o valor calculado foi ponderado em $70 \%$ de seu valor, tendo como valor final de $0,345 \mathrm{~mm}$. O fator $\mathrm{k}$ é definido como 1,14 como indicam Taitel e Dukler (1976) e o cálculo do fator de atrito é realizado utilizando a equação (4).

$$
\begin{aligned}
& \mathrm{d}_{\text {máx }}=\mathrm{k}(\sigma / \rho \mathrm{l})^{3 / 5}\left(2 * \mathrm{fLM}^{*} \mathrm{Vm}^{3} / \mathrm{Di}_{\mathrm{i}}\right)^{-2 / 5} \\
& \mathrm{fLM}=0,046\left(\rho 1^{*} \mathrm{Vm} * \mathrm{Di} / \mu \mathrm{l}\right)^{-0,2}
\end{aligned}
$$

Sendo Di o diâmetro interno do tubo, flm é o fator de atrito de Blasius, vm a velocidade da mistura, $\rho 1$ a massa específica do líquido, $\mu$ f a viscosidade dinâmica do líquido e $\sigma$ é a tensão superficial da água.

Para o estudo do escoamento no estado permanente varias malhas foram geradas no ICEMANSYS. A definição da geometria da malha foi feita após várias tentativas de construções da malha numérica. Para a escolha da malha foi levado em consideração à interferência da blocagem e dos números de elementos nos resultados do processo. A malha numérica utilizada na simulação do estado permanente, após vários refinamentos, é constituída de aproximadamente 345 mil elementos hexaédricos. Processo semelhante foi utilizado para definir a malha para a simulação no estado transiente, sendo escolhida uma malha de aproximadamente 135 mil elementos. Os resultados com os testes para escolha da malha foram satisfatórios, porém não serão mostrados por não fazer parte do escopo deste trabalho.

Para o estado permanente a vazão mássica utilizada na entrada da tubulação é de $1,43 \mathrm{~kg} / \mathrm{s}$. Pressão na saída de 4,5 bar e nos furos de 0 bar. O processo computacional demorou cerca de 13 horas com um total de 720 interações. Para o estado transiente a velocidade de entrada da água foi de $5,02 \mathrm{~m} / \mathrm{s}$ e a do ar de 2,036 m/s, como parâmetro de inicialização a velocidade na entrada foi definida como a soma destas duas velocidades. A pressão na inicialização, na saída e nos furos foi de 0 bar. Para o processo transiente, os cálculos computacionais demoraram cerca de 1 hora e 40 min para convergência. $\mathrm{O}$ tempo total de escoamento foi $5 \mathrm{~s}$, com passo de tempo de $0.05 \mathrm{~s}$. Os tempos considerados para análise das variáveis foi 0,05; 0,6 e $5 \mathrm{~s}$. Para ambas as condições (estacionário e transiente), foram consideradas: Sistema isotérmico a $25{ }^{\circ} \mathrm{C}$, sem padrão de empuxo, modelo de turbulência k-epsilon padrão, turbulência sendo definida como média, parede lisa, modelo de Partícula, força de arrasto pelo método de Schiller Naumann, as forças de interface não foram consideradas, a transferência de massa e turbulência não foram consideradas, esquema de advecção e de turbulência foram respondidos pelo modelo high resolution, e erro padrão. Dessa forma, o tubo a 
ser simulado possui $20 \mathrm{~m}$ de comprimento, 3/4" de diâmetro e um furo circular de $0,1 \mathrm{~cm}$ de raio, representando o vazamento. Os furos estão localizados em 8 e 12 m, contado a partir do início.

O perfil do escoamento foi analisado em seções longitudinais da tubulação, em duas regiões distintas: no topo da tubulação e na região central. Para interpretação do programa, isto representa passar uma linha no centro da tubulação e outra linha na parte superior da tubulação onde se localizam os vazamentos a fim de se adquirir os dados necessários.

\section{RESULTADOS E DISCUSSÕES}

O tema proposto neste trabalho abordou um estudo de um problema comum no transporte de fluidos através de tubulações. Neste tipo de transporte, a detecção de vazamentos muito pequenos é bastante difícil com a instrumentação disponível (Tavares, 2012).

A Figura 1a representa o gráfico do perfil da pressão ao longo do tubo para o estado estacionário nas duas regiões estudadas. Na parte superior da tubulação, podem-se ver as perturbações características de um vazamento, ou seja, o decréscimo e logo em seguida o re-estabelecimento da pressão em outro patamar. Já na parte central esse comportamento é imperceptível e sua detecção já não seria possível.
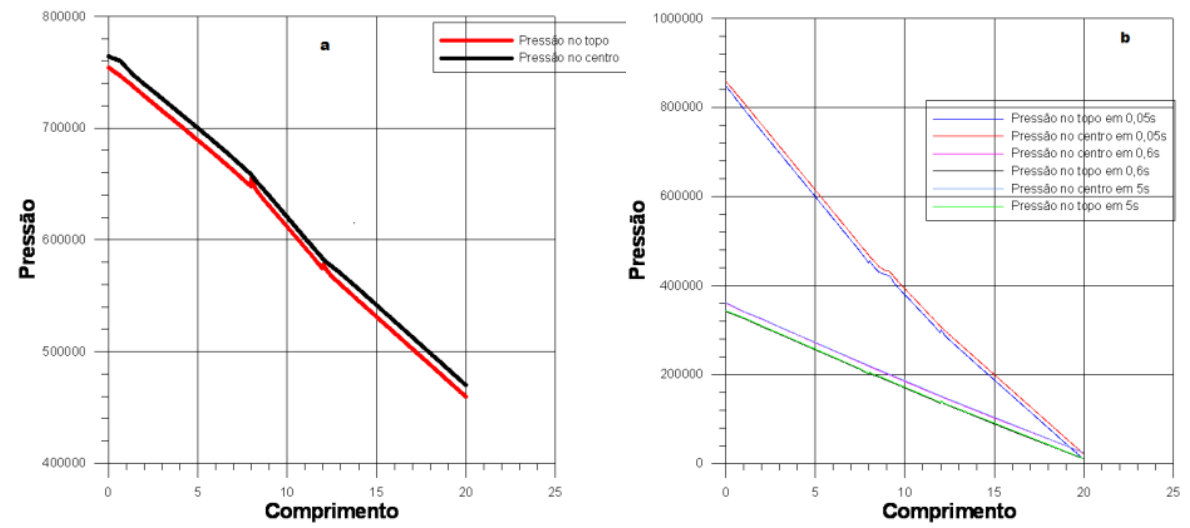

Figura 1 - Gráfico da pressão ao longo do tubo nas regiões de estudo no estado permanente (a) e no estado transiente (b).

A Figura $1 b$ representa o comportamento da pressão ao longo do tubo no estado transiente. Nos primeiros instantes após o vazamento, $\mathrm{t}=0,05$; percebe-se que a pressão apresenta um comportamento variante nos pontos de vazamento e entre eles. Segundos depois, 0,6s; re-estabelece em um novo patamar ao se estabilizar a nova perda de carga no sistema. Pode-se observar ainda que sua estabilização é rápida pela pouca variação do perfil em 5s com relação a 0,6s.

A Figura 2 mostra o perfil da velocidade superficial e real da água e do ar nas duas regiões estudadas no estado estacionário ao longo da tubulação. Pode-se observar que as velocidades superficiais e reais da água tende a se igualarem após o comprimento de aproximadamente $15 \mathrm{~m}$ para as duas situações estudadas. Como a massa específica do ar é menor do que a da água, e o furo 
estando situado na parte superior do tubo, o ar tende a escoar com mais facilidade, logo, com o vazamento, uma maior quantidade de ar é perdida e sua fração tende à zero, como pode ser observado. A velocidade superficial do ar tende a zero pelo fato de que a fração volumétrica de ar tende a zero, como pode ser observado na Figura 3. No entanto a velocidade real do ar sofre uma diminuição pela perda do fluido no sistema decorrente dos vazamentos alcançando um novo valor. É perceptível também que em um mesmo ponto a velocidade real da água não é igual a velocidade real do ar, o que é esperado, pois os fluidos por possuírem propriedades diferentes movem-se com diferentes velocidades.

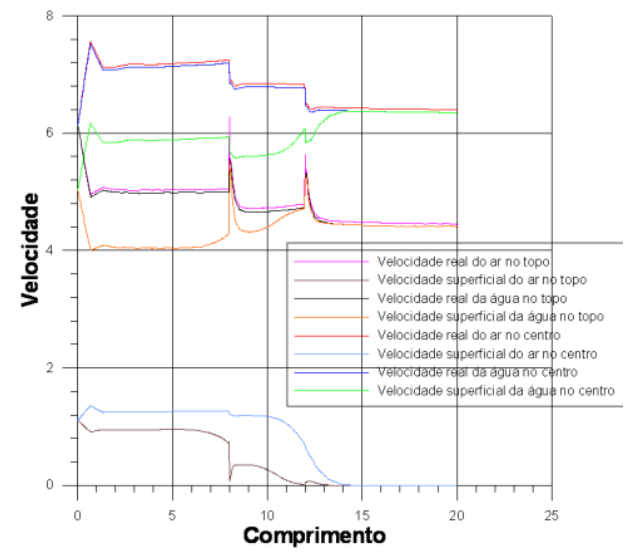

Figura 2 - Gráfico da velocidade superficial e real da água e do ar nas regiões de estudo.

A figura 3 mostra o comportamento da fração volumétrica da água nas duas regiões estudadas ao longo da tubulação. Este comportamento comprova o que foi dito anteriormente, uma vez que a fração molar da água atinge o seu valor máximo igual à unidade.

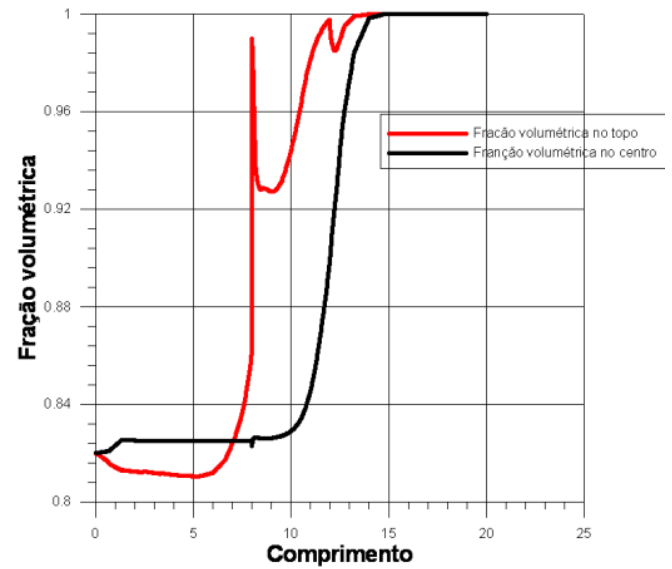

Figura 3 - Gráfico da fração volumétrica da água nas regiões de estudo.

A Figura 4 representa a fração volumétrica da água no estado transiente ao longo da tubulação. O gráfico mostra que por decorrência dos vazamentos há um grande aumento da fração volumétrica 
da água uma vez que a fração do ar diminui, e sua estabilização, após aproximadamente 2s, ocorre com a fração volumétrica da água próxima da unidade.
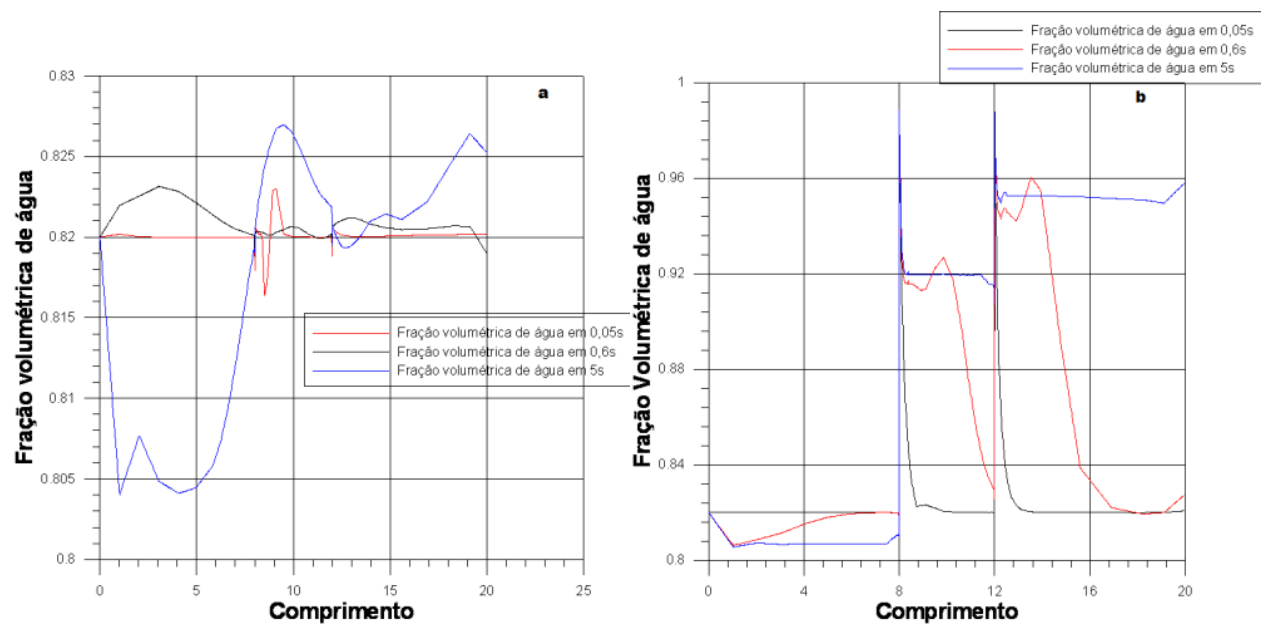

Figura 4 - Gráficos da fração volumétrica da água no centro (a) e no topo (b) no estado transiente.

As Figuras 5 e 6 mostram o comportamento das velocidades para o estado transiente. Na Figura 5, em 0,05s; percebe-se a queda nas velocidades do ar e da água nas imediações do vazamento, se estabelecendo ao longo do tubo. Para o instante de 0,6s, Figura 6a, observa-se que um novo perfil de velocidade é alcançado, que apresenta velocidades ligeiramente maiores do que no instante anterior decorrente do acréscimo da vazão necessária para manter as condições pré-estabelecidas. Decorrente do fato de que a água se apresenta em maior quantidade, os valores de velocidade superficial e real da água se aproximam após os vazamentos. A recíproca acontece para o ar, tendo então sua velocidade superficial um valor baixo, porém mantendo uma velocidade real considerável. É ainda possível notar que as velocidades reais da água e do ar não apresentam o mesmo valor na mesma posição e instante, como esperado.

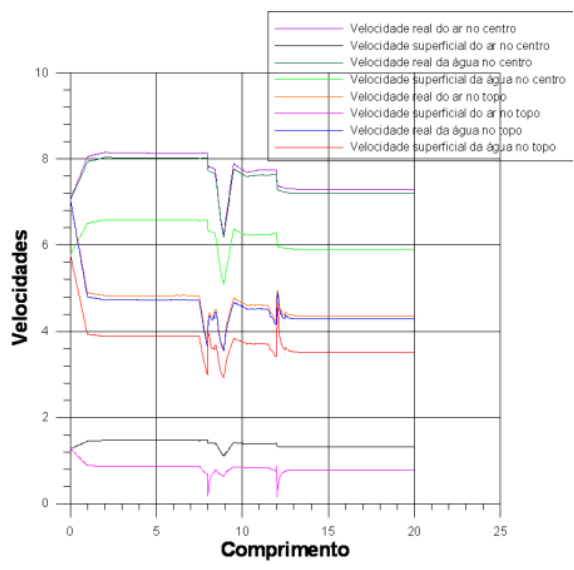

Figura 5 - Gráfico da velocidade superficial e real da água e do ar nas regiões de estudo em 0,05s. 

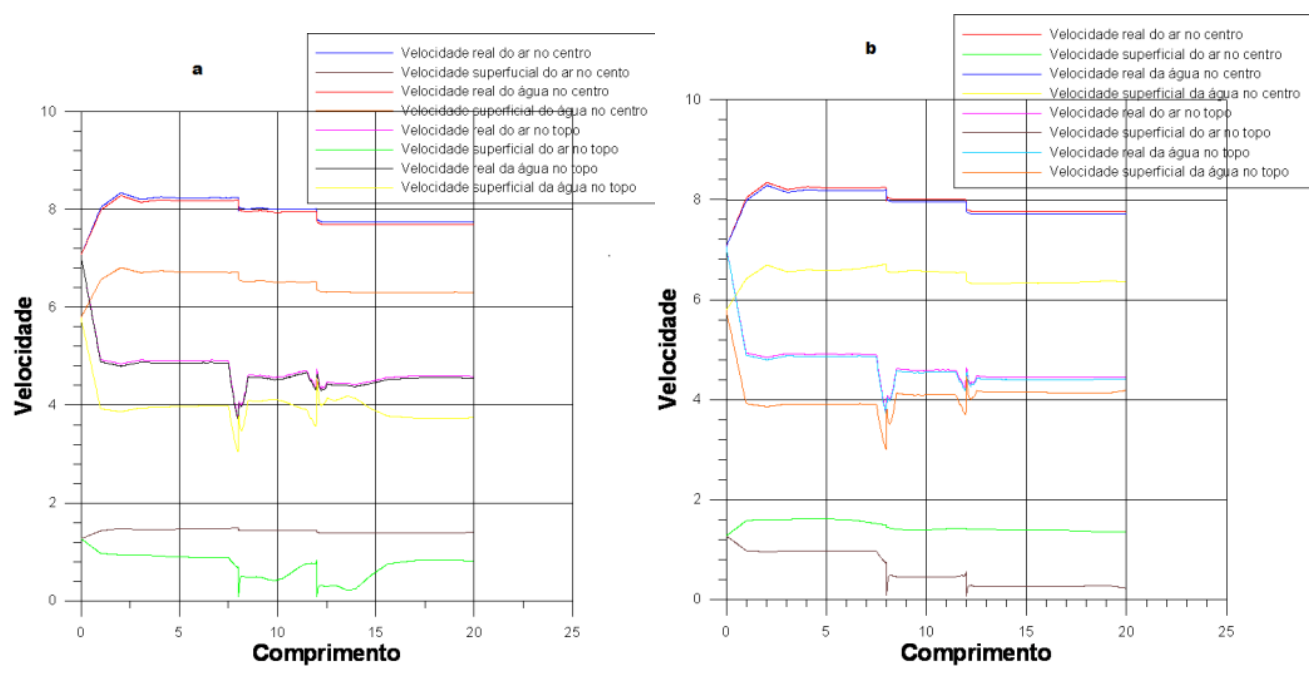

Figura 6 - Gráfico da velocidade superficial e real da água e do ar nas regiões de estudo em (a) 0,6 s e (b) $5 \mathrm{~s}$.

\section{CONCLUSÕES E OBSERVAÇÕES}

Com o auxílio dos gráficos e algumas ferramentas disponibilizadas pelo software ANSYS-CFX foi possível analisar cada variável e seu comportamento no decorrer do tempo e espaço podendo obter uma análise qualitativa adequada do processo. Tanto no estacionário quanto no transiente a velocidade real da água tende a se equiparar a velocidade superficial da água pelo fato de que a água torna-se quase uma fase pura na presença do vazamento. Para o ar, a velocidade superficial tende a zero pelo fato de que sua fração tende a zero, tendo uma pequena vazão em uma grande área transversal total. A velocidade real se comporta da mesma forma que para a água, tendo um valor considerável. Para o estado transiente sua estabilização acontece próximo de 2,5 s.

Os resultados foram satisfatórios visto que o perfil do escoamento está de acordo com o esperado, baseado nas características descritas na literatura para o estudo, levando em consideração as considerações adotadas.

\section{REFERÊNCIAS}

Baker, O., 1954. Design of pipelines for simultaneous flow of oil and gas. Oil Gas J., 53, pp. 185195;

Bakker, A. Applied Computational Fluid Dynamics. Fluent Inc., Leitura 14, 2002. Disponível em: <http://www.bakker.org/dartmouth06/engs150/01-intro.pdf> . Acesso em: 14/04/2014;

Bigarella, E. D. V. Verificação e Validação em CFD. Mesa Redonda em Modelagem - Rede Nacional de Combustão, Embraer, 2007;

Colombo, A. F.; LEE, P.; KARNEY, B. W. A Selective Literature Review of Transient-Based Leak Detection Methods. Journal of Hydro-Environment Research, vol. 2, n. 4, p. 212-227, 2009; 
Hinze, J. O, 1955. Fundamentals of the hydrodynamic mechanism of splitting in dispersion process. AIChE J., 1, pp. 289;

KORKISCHKO, I. Investigação Experimental e Simulação Numérica do Escoamento ao Redor de um Modelo Automobilístico: Corpo de Ahmed. 2006. 100 p. Monografia (Engenharia Mecânica) - Escola Politécnica da Universidade de São Paulo, São Paulo, SP, 2006;

Lockhart, R.W. e Martinelli, R.C., Proposed Correlation of Data for Isothermal Two-Phase, Two Component Flow in Pipes, Chemical Engeneering Progress - Vol. 45, 1949;

LOMAX, H.; PULliAM, T. H.; ZINGG, D. W. Fundamentals of Computational Fluid Dynamics. NASA Ames Research Center and University of Toronto Institute for Aerospace Studies. August 26, 1999. Disponível

em: <http://maji.utsi.edu/courses/07_681_advanced_viscous_flow/ref_af6_Fundamentals_of_CFD.pdf> Acesso em: 09/04/2014;

MARINHO, J. L. G. Estudo do Escoamento Multifásico Tipo Bolha de Taylor em Dutos e Conexões Curvadas. 2008. Dissertação (Mestrado em Engenharia Química) - Universidade Federal de Campina Grande, Campina Grande, PB, 2008;

PHMSA - U.S. Department of Transportation Pipeline and Hazardous Materials Safety Administration. Significant Pipeline Incidents, 2010;

SOUZA, J. N. M. Modelagem e Simulação de Escoamento Multifásico em Dutos de Produção de Óleo e Gás Natural. 2010. Tese (Doutorado em Tecnologia de Processos Químicos e Bioquímicos) Universidade Federal do Rio de Janeiro, Escola de Química, Rio de Janeiro, RJ, 2010;

Taitel, Y. and Dukler, A.E., 1976. A model for predicting flow regime transition in horizontal and near horizontal gas-liquid flow. AIChE Journal, 22, pp. 47- 55;

Tavares, D. P. S. de A., Escoamento Trifásico Não-Isotérmico em Oleodutos Horizontais com Vazamentos: Modelagem e Simulação. 2012. 192f. Dissertação - UNIVERSIDADE FEDERAL DE CAMPINA GRANDE, Paraíba. 2012;

Tilton, J.N. Fluid and Particle Dynamics. McGraw-Hill, Perry's Chemical Engeneers' Handbook, $8^{\text {th }}$ Ed. The McGraw-Hill Companies. 